\title{
Papel das transferências intergovernamentais na equalização fiscal dos municípios brasileiros
}

\author{
Alexandre Lima Baião \\ Fundação Getúlio Vargas (FGV) \\ Armando Santos Moreira da Cunha \\ Fundação Getúlio Vargas (FGV)
}

Flávio Sergio Rezende Nunes de Souza

Diretoria de Administração da Marinha (DAdM)

Diante da heterogeneidade econômica e social dos municípios brasileiros, tornase fundamental estudar a distribuição dos recursos das transferências, verificando se os municípios que mais necessitam de suporte financeiro têm sido os efetivamente beneficiados. Este trabalho busca avaliar o efeito das transferências intergovernamentais na equalização fiscal dos municípios, investigando se os diversos tipos de transferências conseguem levar em consideração a capacidade de autofinanciamento do município e a necessidade fiscal atrelada a custos e demandas por serviço público. Os resultados evidenciam que algumas transferências, que têm natureza redistributiva, como o Fundo de Participação Municipal (FPM), contribuem pouco para a equalização, enquanto que algumas transferências condicionais, como o Fundeb, que não possuem esse objetivo, apresentam um efeito positivo. As transferências voluntárias são relativamente neutras, provavelmente por não seguirem critérios, nem econômicos, nem redistributivos, mas políticos. O Bolsa Família, apesar de não ser uma transferência intergovernamental, atenua as distorções realizadas pelas demais transferências observadas.

Palavras-Chave: política fiscal, transferência intergovernamental, orçamento fiscal, administração municipal

[Artigo recebido em 12 de maio de 2016. Aprovado em 7 de abril de 2017.] 


\section{Papel de las transferencias intergubernamentales en la ecualización fiscal de los municipios brasileños}

Dada la heterogeneidad económica y social de los municipios brasileños, es esencial estudiar la distribución de las transferencias de fondos, asegurándose de que los municipios con mayor necesidad de apoyo financiero han sido los beneficiarios de manera efectiva. Este trabajo tiene como objetivo evaluar el efecto de las transferencias intergubernamentales en la compensación fiscal de los municipios, investigando si los diversos tipos de transferencias tienen en cuenta la capacidad de autofinanciación del municipio y las necesidades fiscales ligadas a los costes y las exigencias de servicio público. Los resultados muestran que algunas transferencias, que tienen la naturaleza distributiva, como el FPM, contribuyen poco a ecualización, mientras que algunas transferencias condicionadas, como Fundeb, que no cuentan con este fin, tienen un efecto positivo. Transferencias voluntarias son relativamente neutral, probablemente por no seguir criterios económicos o de redistribución, sino políticos. El Bolsa Familia, aunque no es una transferencia intergubernamental, mitiga las distorsiones hechas por otras transferencias observadas.

Palabras clave: política fiscal, transferencia intergubernamental, presupuesto fiscal, administración municipal

\section{The role of intergovernmental transfers in fiscal equalization of Brazilian municipalities}

Given the economic and social heterogeneity of Brazilian municipalities, it is essential to study the distribution of resources transfers, verifying if the municipalities that most need financial support have been the effective beneficiaries. This study investigates the effect of intergovernmental transfers on fiscal equalization, investigating whether the different types of transfers can take into account the self-financing capacity of the municipality and the fiscal necessity related to costs and demands for public service. The results show that some transfers as the FPM contribute little to fiscal equalization. On the other hand, some conditional transfers, as Fundeb, in spite of not being focused on horizontal disparities mitigation, have a positive effect on fiscal equalization. Voluntary transfers are relatively neutral, probably because they follow neither economic, nor redistributive criteria, but just address political purposes instead. Bolsa Família, although not being an intergovernmental transfer, can mitigate the distortions created by other transfers.

Keywords: fiscal policy, intergovernmental transfer, fiscal budget, municipal administration 


\section{Introdução}

A tendência de descentralização na administração pública que se verificou nas últimas décadas criou um desafio para os países organizados em regimes federativos. Os governos subnacionais receberam maiores atribuições, assim passaram a ser responsáveis pela aplicação de um maior volume de recursos na provisão dos serviços públicos e a ter maior participação na implementação das políticas públicas. No entanto, alguns fatores, como a necessidade de manter a eficiência do sistema tributário e de garantir a aplicação ótima de recursos no nível subnacional em determinados setores, fazem com que a arrecadação se mantenha centralizada. Assim, em todas as federações do mundo, a arrecadação é maior no nível mais amplo e menor nos níveis locais, tornando necessária a transferência de recursos da instância central aos governos subnacionais (REZENDE, 2006). Essas transferências, além de corrigirem o desequilíbrio vertical na federação, ou seja, as diferenças entre atribuições e receitas nos diferentes níveis de governo, representam uma oportunidade de atenuar as disparidades regionais (BACHUR, 2005; BATISTA, 2015).

No Brasil, essas transferências tornam-se ainda mais importantes, devido à grande heterogeneidade regional e à extensão do território brasileiro. Conforme destaca Rezende (2006), um dos desafios do federalismo fiscal brasileiro é enfrentar a ampliação das disparidades regionais, que concentram as bases tributárias em pontos específicos do território, e, consequentemente, afetam a repartição das receitas tributárias entre os entes federativos.

Os indicadores sociais também revelam um quadro heterogêneo, em que os indivíduos situados em determinadas regiões recebem serviços públicos e oportunidades profissionais muito distintas da média brasileira. O Sudeste, por exemplo, apresenta uma renda domiciliar per capita mais de duas vezes superior à média do Nordeste, segundo os dados do censo demográfico de 2010. Outro exemplo é a taxa de analfabetismo da população de 15 anos ou mais, que é de 24,3\% na região do semiárido, enquanto a média nacional é de 9,6\% (INSTITUTO BRASILEIRO DE GEOGRAFIA E ESTATísticA, 2010).

Nesse sentido, a literatura aponta a importância das transferências para atenuar as desigualdades regionais por meio da equalização fiscal, beneficiando municípios com menor base tributária e maiores custos de provisão dos serviços públicos. Essas transferências buscam tornar a provisão dos serviços públicos mais equitativa, permitindo que os governos locais, para dado nível de esforço fiscal, ofereçam o mesmo volume e qualidade de serviços à população (BATISTA, 2015; REZENDE, 2010; WANG; HERD, 2013). 
As transferências redistributivas devem incorporar, num cenário ideal, duas informações básicas: a necessidade e capacidade fiscal de cada ente federativo. É natural, por exemplo, que municípios com uma grande parcela da população em idade escolar apresentem maiores despesas com serviços públicos de educação, bem como municípios com maior atividade econômica tenham maior capacidade de captar recursos por meio da arrecadação.

Porém, é comum que apenas uma parte das transferências se insira na proposta de equalização fiscal, pois é importante preservar a eficiência econômica a nível nacional. De fato, a literatura aponta um trade-off entre eficiência e equidade no planejamento das transferências intergovernamentais. Dada a necessidade de apresentar ambos os tipos de repasse, é desejável que as transferências devolutivas tenham critérios eficazes para cumprir seu papel, bem como as transferências equalizadoras utilizem parâmetros eficazes para realizar a equalização. Não há muita discussão sobre a eficácia dos critérios devolutivos, mas muitos autores apontam problemas em transferências que, supostamente, deveriam combater as desigualdades regionais, mas acabam beneficiando ou prejudicando determinadas localidades sem nenhum fundamento lógico que as sustentem (MENDES; MIRANDA; Cossio, 2008; Prado, 2001, 2007).

As transferências representam a maior fonte de receita orçamentária dos municípios brasileiros. Contudo, apesar da dimensão dos recursos públicos que são repassados aos governos municipais, ainda há poucosestudos que investiguem de forma sistemática os efeitos dessas transferências na redistribuição e redução do hiato fiscal. O estudo encontrado que mais se aproxima dessa proposta foi elaborado por Mendes, Miranda e Cossio (2008), em que os autores avaliaram cada uma das principais transferências intergovernamentais em diversos quesitos, como autonomia, redistribuição regional e accountability. O hiato fiscal (fiscal gap), que é o foco do presente trabalho, é analisado de forma separada para cada transferência. O presente estudo vai além, analisando o conjunto agregado dos fluxos de recursos destinados aos municípios, pois um repasse pode contrabalancear o efeito de outro, e, portanto, é importante não limitar o estudo à investigação individual de cada transferência (SCHROEDER; SMOKE, 2003). Além do mais, os autores analisaram cada indicador de capacidade fiscal e necessidade fiscal separadamente, ou seja, por meio de uma perspectiva bivariada, enquanto este trabalho busca fazer esta análise conjunta, por meio da regressão linear.

Dessa forma, esta pesquisa visa identificar quais transferências intergovernamentais estão sendo distribuídas de acordo com a capacidade e necessidade fiscal dos municípios brasileiros. 


\section{Referencial teórico}

\section{Transferências intergovernamentais}

Apesar da tendência de descentralização que pode ser verificada nos diversos países nas últimas décadas (ABRúcıo, 2005), todas as federações ainda centralizam em algum grau sua arrecadação tributária. Segundo Prado (2007), em todas as federações do mundo, os governos dos níveis superiores arrecadam mais do que gastam, enquanto os de níveis inferiores gastam mais do que arrecadam. Esse fenômeno recebe o nome de brecha vertical e explica por que todas as federações fazem uso de transferências verticais. Nesse sentido, esse autor aponta que existem três fatores que justificam a existência de algum grau de centralização da arrecadação nas federações.

O primeiro fator é a necessidade de garantir a eficiência do sistema tributário. Centralizar alguns impostos reduz o custo de atender diversas legislações (compliance costs) incorrido pelos agentes econômicos (MENDES; MIRANDA; CossIO, 2008; PRADO, 2001) e diminui o risco de governos subnacionais ferirem o princípio da neutralidade ou até realizarem competições fiscais, à medida que alteram suas alíquotas (PRADO, 2006).

O segundo fator é a necessidade de promover a equidade entre os governos subnacionais, que geralmente apresentam capacidades de arrecadação diferentes, bem como demandas da população e respectivos custos de atendimento distintos. Essa diferença recebe o nome de brecha horizontal e representa o motivo das transferências distributivas, que visam atenuar a desigualdade entre estados e municípios. Essa análise também se estende à relação entre os governos centrais e subnacionais, pois, com a divisão das funções e competências tributárias entre os entes, é natural que exista um desajuste no volume de recursos que cada nível federativo consegue arrecadar e no custo das atividades pelas quais ele é responsável. A essa disparidade, a literatura dá o nome de brecha vertical. Dessa forma, as transferências se justificam na medida em que as brechas horizontais e verticais precisam ser corrigidas (SCHROEDER; SMOKE, 2003).

Oterceiro fator éa exigência de alocar os recursos de maneira seletiva e discricionária a fim de realizar projetos e objetivos nacionais. Nesse caso, impõem-se condições para o uso do dinheiro, o que permite controlar o alcance dos resultados do projeto. Vale ressaltar que, nesse caso, as transferências condicionadas criam resistência entre os governos subnacionais, que passam a ter sua autonomia restringida.

A literatura nacional e internacional apresenta várias alternativas de classificação das transferências intergovernamentais. Alguns dos critérios utilizados referem-se 
a: a) como é determinado o volume total de recursos a ser distribuído aos governos subnacionais; b) como são distribuídos os recursos entre os governos; c) existência ou não de restrições sobre como o recurso pode ser aplicado (transferências condicionais); d) existência ou não de contrapartida financeira do governo receptor (matching ou non-matching grants) (BLÖCHLIGER, 2013; CYRENNE; PANDEY, 2015).

Apesar de ser possível definir diversas combinações a partir desses critérios, nem todos os possíveis tipos de transferências formadas pelas combinações são encontrados nas federações. Além do mais, cada federação utiliza padrões diferentes de transferências, tornando relevante utilizar uma classificação adaptada para o caso do Brasil. Nesse sentido, Mendes, Miranda e Cossio (2008) propõem um recorte analítico para a Federação brasileira, agrupando as transferências em seis grupos: a) transferências incondicionais redistributivas; b) transferências incondicionais devolutivas; c) transferências condicionais voluntárias; d) transferências condicionais obrigatórias; e) transferências ao setor produtivo privado; f) transferências diretas a indivíduos.

As transferências incondicionais redistributivas são distribuídas segundo fórmulas que incluem indicadores demográficos e socioeconômicos, buscando corrigir as brechas horizontais e verticais. Por serem incondicionais, não apresentam nenhuma restrição sobre a decisão de aplicação pelo governo local. Os principais exemplos são o Fundo de Participação Estadual (FPE) e o Fundo de Participação Municipal (FPM).

As transferências incondicionais devolutivas distribuem os recursos para os governos em que foi realizada a arrecadação, sem a presença de condicionalidades sobre a forma de gasto. Um exemplo é o Imposto sobre Circulação de Mercadorias e Prestação de Serviços (ICMS), em que no mínimo 75\% é distribuído de acordo com o valor adicionado fiscal (VAF) dos municípios. O VAF é uma proxy da base tributária do ICMS e indica quanto foi arrecadado do imposto no município.

As transferências condicionais voluntárias são representadas pelos acordos e convênios, em que o governo central concede recursos para governos subnacionais com a condição de que determinadas atividades sejam realizadas. Nesse caso, o governo central tem certo grau de discricionariedade para decidir sobre a transferência.

As transferências condicionais obrigatórias correspondem a recursos vinculados distribuídos de acordo com critérios não devolutivos. Exemplos importantes são as transferências do Sistema Único de Saúde (SUS) e do Fundo Nacional de Desenvolvimento e Manutenção da Educação Básica (Fundeb). Essas transferências visam garantir o investimento adequado em setores que poderiam receber atenção insuficiente pelo governo local, devido à presença de externalidades. 
Existem ainda transferências que não são intergovernamentais, mas que interferem no equilíbrio federativo e na redistribuição regional da renda. As transferências ao setor produtivo privado buscam subsidiar empreendimentos privados em regiões menos desenvolvidas. As transferências diretas a indivíduos são recursos distribuídos diretamente para parcela da população em situação de vulnerabilidade, como é o caso do Programa Bolsa Família.

\section{Equalização fiscal}

Dado o papel das transferências na promoção da equidade da provisão dos serviços públicos e, em determinadas situações, na defesa da eficiência alocativa, torna-se necessário saber como definir quanto cada governo local irá receber do recurso. A literatura aponta dois aspectos principais que devem ser levados em consideração na equalização horizontal: a necessidade fiscal e a capacidade fiscal (BATISTA, 2015; MARTINeZ-VAsquez; BoeX, 1999).

A capacidade fiscal expressa quanto o governo subnacional consegue arrecadar para determinado nível de esforço fiscal, refletindo a base tributária de sua localidade e a renda de sua população. Espera-se, por exemplo, que municípios com maior produto interno bruto (PIB) e renda per capita consigam arrecadar maiores volumes de recursos comparados a municípios menos dinâmicos economicamente.

Já a necessidade fiscal incorpora as diferenças entre o nível de serviços públicos necessários para cada localidade, devido às características da população ou da região. Um exemplo é o de municípios que, devido a uma maior parcela de sua população estar em idade escolar, necessitam aplicar mais no setor de educação.

Os sistemas de equalização fiscal devem ser capazes de isolar, entre os fatores que levam a uma base tributária menor ou a uma alta necessidade de custeio, aqueles que estão sob controle do governo local daqueles que lhe são externos. Caso a situação fiscal do município seja decorrência de fatores controláveis pelo governo, como a má gestão dos recursos, por exemplo, a compensação realizada por meio das transferências implicaria a criação de incentivos prejudiciais ao desempenho dos governos locais (CYRENNE; PANDEY, 2015; DAFFLON, 2007).

Assim, o sistema de equalização fiscal ideal deveria distribuir os recursos de forma inversamente proporcional à capacidade fiscal dos municípios, e de forma proporcional às suas necessidades fiscais. Martinez-Vasquez e Boex (1999) explicam que o modelo mais sofisticado consegue definir o valor do repasse ao governo subnacional de acordo com seu hiato fiscal, ou seja, pela diferença entre suas necessidades fiscais e sua capacidade de obtenção dos recursos via os tributos de sua competência. Atualmente um dos sistemas mais complexos é o da Austrália, que utiliza tanto a informação da capacidade de arrecadação quanto da necessidade fiscal de cada governo subnacional. 
Mais especificamente, há duas abordagens principais utilizadas para definir a distribuição dos recursos no contexto da equalização fiscal: os sistemas de indicadores macro e os sistemas representativos. Os primeiros utilizam indicadores demográficos ou econômicos que refletem a capacidade fiscal ou a necessidade fiscal do município, como a renda per capita, a população ou o PIB municipal. Já os sistemas representativos identificam, no caso da capacidade fiscal, quanto cada jurisdição obteria de recursos se aplicasse os valores médios de taxas tributárias utilizados na federação, e, no caso da necessidade fiscal, o gasto per capita necessário para prover um conjunto padronizado de serviços aos munícipes.

No caso da capacidade fiscal, o sistema representativo é denominado de Representative Tax System (RTS). No RTS, primeiramente se identifica a prática adotada ao longo da federação, como as bases tributárias utilizadas e as taxas médias aplicadas de tributação (BOADWAY, 2007). A partir desses valores, é calculado, para cada governo subnacional, o valor que ele obteria caso utilizasse essa prática padrão.

Ainda dentro dos sistemas representativos, a abordagem que equaliza os gastos do governo local inclui diferentes métodos, que buscam identificar diferenças em fatores que afetam a despesa local, como diferenças na demografia, na geografia, nas necessidades locais e nas políticas públicas, definindo o mínimo necessário que cada município precisa receber para oferecer um serviço padrão (RESCHOVSKY, 2007).

Outra alternativa é o uso de medidas macro que expressem as demandas, os custos e a capacidade de gerar receita própria do governo local. Essa metodologia é amplamente utilizada pelas federações por representar uma alternativa mais simples, transparente e que requer menos dados. Vale ressaltar que, nas nações em que os governos locais têm menos autonomia para determinar o nível dos tributos, proxies da base tributária podem se mostrar suficientes para identificar a capacidade ou a necessidade fiscal. Da mesma forma, a disponibilidade reduzida de dados que são exigidos nos sistemas mais sofisticados, principalmente em países em desenvolvimento, leva os governos a optar por essa abordagem macroeconômica (WILSON, 2007).

Contudo, a equalização fiscal não é uma panaceia e acarreta também um ônus, na medida em que transferir recursos de regiões mais desenvolvidas para regiões mais vulneráveis pode reduzir o crescimento econômico a nível nacional. Um possível exemplo desse efeito pode ser encontrado na Alemanha. Rezende (2006) explica que existem dúvidas sobre a viabilidade a longo prazo do "federalismo 
cooperativo" alemão, pois a transferência de recursos dos estados mais ricos para os mais pobres estaria gerando incentivos equivocados, segundo alguns especialistas, comprometendo a economia como um todo. Já na distribuição e alocação economicamente eficiente de recursos nas diferentes regiões do país, determinada, não pelos propósitos redistributivos, mas sim pela capacidade local de arrecadação, ligada diretamente à atividade econômica regional, a economia nacional é favorecida enquanto a equidade é sacrificada. Portanto, em algumas situações existe um trade-off entre promover o crescimento econômico nacional e reduzir as desigualdades (CYREnNe; PANDEY, 2015; MACIEL; ANDRADE; TELLES, 2008).

Nem sempre esse trade-off se faz presente, pois, conforme destacam Schroeder e Smoke (2003), é importante identificar os motivos que levam determinada região a estar menos desenvolvida. Se sua situação reflete a ausência de um nível mínimo de fatores de produção, as transferências redistributivas podem representar uma medida ineficaz. Por outro lado, se as condições dessa área derivarem de uma trajetória histórica marcada pela ausência do poder público e pelo baixo nível de investimento estatal, a equalização pode promover, simultaneamente, equidade e eficiência econômica.

Ainda, as diferenças nas necessidades e capacidades fiscais, se não forem corrigidas por transferências equalizadoras, podem prejudicar a eficiência econômica ao fomentar fluxos migratórios contraproducentes. A disparidade no benefício líquido fiscal (net fiscal benefits) oferecido por diferentes jurisdições, ou seja, a diferença entre o valor (utilidade) recebido pelo cidadão por meio dos serviços públicos consumidos, deduzido do ônus fiscal absorvido por ele, pode incentivar movimentos migratórios avessos à racionalidade produtiva (CYRENNE; PANDEY, 2015). Nesse caso, se ganhos idênticos podem ser obtidos em duas regiões, o munícipe provavelmente irá preferir residir naquela com maior benefício fiscal líquido, causando uma alocação ineficiente do trabalho no território. Se o indivíduo preferir continuar em sua região, apesar de sofrer uma maior carga tributária, ou menor consumo de serviços públicos, as diferenças tributárias estarão gerando uma situação de inequidade entre cidadãos (BOADWAY, 2007).

\section{Metodologia}

\section{Especificação do modelo}

A literatura explica que o município deve receber recursos, no âmbito da equalização fiscal, de acordo com o gap entre sua capacidade de arrecadação e 
suas necessidades fiscais. Diante disso, este trabalho analisou o quadro geral das transferências intergovernamentais, em especial as redistributivas, buscando identificar aquelas que efetivamente promovem a equalização fiscal.

No campo das transferências redistributivas brasileiras, coexistem uma série de critérios e fundos que buscam reduzir a desigualdade regional. Essas transferências intergovernamentais não consideram umas às outras, mas consistem em fluxos independentes e isolados (PRADo, 2001). Nesse sentido, uma transferência pode suavizar ou até anular o efeito da outra. Um exemplo é o FPM, que beneficia municípios menos populosos, enquanto a parcela redistributiva do ICMS, em alguns estados, beneficia municípios com população maior. Por esse motivo, é necessário realizar uma análise que contemple a totalidade das transferências, em vez de investigar apenas um dos fundos ou repasses (SCHROEDER; SMOKE, 2003). Este trabalho segue esse direcionamento, analisando cada repasse individualmente, concomitante à análise do quadro global de transferências.

Mais especificamente, este trabalho utiliza a metodologia proposta por Martinez-Vasquez e Boex (1999), em que se identifica por meio de uma regressão linear, como o valor da transferência varia em relação a mudanças em indicadores da capacidade econômica e da necessidade fiscal do município. Essa metodologia foi escolhida por ter sido elaborada justamente visando sua aplicação em países em desenvolvimento, com pouca disponibilidade de dados.

Dessa forma, neste trabalho realiza-se uma regressão do valor total das transferências legais contra as variáveis representativas da capacidade fiscal renda domiciliar per capita, PIB municipal per capita - e da necessidade fiscal crescimento populacional nos anos recentes, percentual da população de 0 a 14 anos, percentual da população com 60 anos ou mais, percentual de pessoas com 15 anos ou mais analfabetas, percentual de população em situação de extrema pobreza e densidade populacional. Para tal, utiliza-se a seguinte equação:

$$
C_{i}=\alpha_{i} \cdot 1+\sum_{k=1}^{K} \beta_{k} x_{k i}+\varepsilon_{i}
$$

Em que $X$ é o valor observado para o indicador de necessidade/capacidade fiscal $k(\mathrm{k}=1,2 . ., \mathrm{K})$ para o município $i$. Ci, por sua vez, representa o valor da transferência.

$\mathrm{C}=$ Transferências intergovernamentais

$\mathrm{X} 1=$ Crescimento populacional

X2 = Percentual da população em situação de extrema pobreza

X3 $=$ Densidade populacional 
X4 = Percentual da população com idade superior a 60 anos

X5 = Percentual da população entre 0 a 14 anos

X6 = Percentual de pessoas com 15 anos ou mais analfabetas

X7 = Renda domiciliar per capita

$\mathrm{X} 8=\mathrm{PIB}$ per capita

Foi realizada mais de uma regressão usando as mesmas variáveis independentes, a fim de estudar diferentes tipos de transferências. Conforme expresso no Quadro 1, em um nível agregado, foram analisadas as transferências legais e voluntárias, separadamente. No âmbito das transferências legais, foram estudados cada um dos principais componentes desse conjunto de repasses. Além disso, mesmo não representando uma transferência intergovernamental, foi incluída na análise a transferência do Programa Bolsa-Família, por representar um fluxo significativo de recursos e por poder afetar, em algum grau, a equalização fiscal.

\section{Quadro 1 - Transferências selecionadas para a análise}

\begin{tabular}{|l|l|}
\hline \multirow{4}{*}{ Transferências legais } & FPM \\
\cline { 2 - 2 } & ICMS \\
\cline { 2 - 2 } & FUNDEB \\
\cline { 2 - 2 } & SUS \\
\cline { 2 - 2 } & Compensação financeira \\
\cline { 2 - 2 } & Outros (não analisado) \\
\hline Transferências voluntárias & \\
\hline Programa Bolsa-Família & \\
\hline
\end{tabular}

Fonte: Elaboração própria.

Para alcançar resíduos mais próximos de uma distribuição normal e, ainda, aumentar a confiabilidade dos resultados com a comparação de modelos complementares, além da regressão com os dados originais, foi realizada, para cada transferência, uma regressão com os dados transformados pela aplicação do logaritmo natural. O modelo sem a transformação foi denominado de M1 e o loglinear de M2.

Este trabalho parte do pressuposto de que uma necessidade fiscal é equalizada quando os municípios que a possuem em maior grau recebem maior volume de recursos, controladas as demais necessidades e a capacidade fiscal. Contudo, discutir se essa relação entre necessidade fiscal e valor da transferência deve ser linear ou logarítmica não está incluído no escopo deste trabalho. Este estudo considera que, se em ambos os modelos a relação foi positiva, há uma evidência suficiente 
de que a equalização da necessidade fiscal tem sido realizada. Se, por outro lado, os sinais do coeficiente são diferentes dependendo da especificação funcional, ou se em um dos modelos o coeficiente não difere significativamente de zero e no outro sim, não há uma convergência dos resultados, e, portanto, inferências sobre o papel da transferência na equalização daquele item devem ser realizadas com precaução. Neste trabalho, opta-se por utilizar as convergências para extrair as principais conclusões e considerar resultados divergentes como objeto de estudos posteriores.

\section{Dados: fontes e tratamento}

Os dados financeiros foram coletados da base Finanças do Brasil: Dados Contábeis dos Municípios (Finbra), referente ao ano de 2010, disponibilizados pela Secretaria do Tesouro Nacional. O PIB municipal utilizado é o de 2009, coletado a partir de pesquisa disponibilizada pelo Instituto Brasileiro de Geografia e Estatística (IBGE). A transferência do Programa Bolsa-família foi obtida por meio da Secretaria de Avaliação e Gestão da Informação (Sagi) do Ministério do Desenvolvimento Social e Combate à Fome. Os demais dados foram obtidos junto ao Censo Demográfico 2010, também realizado pelo IBGE.

A pesquisa contou com dados em seção cruzada (cross section) para 5565 municípios brasileiros. Foram eliminados da análise 354 municípios sem dados financeiros disponíveis. Foram retirados, ainda, 20 municípios com dados insuficientes para um grande número de variáveis financeiras, ou inconsistentes, devido a valores nulos de FPM e ICMS. A análise foi realizada a partir dos 5191 municípios restantes. Essas fragilidades nos dados decorrem do fato de que nem todos os governos locais prestam contas ao Tesouro Nacional, que consolida os dados orçamentário-contábeis do setor público (ORAIR, 2010).

\section{Resultados}

\section{Análise preliminar das variáveis e dos dados utilizados nos modelos}

Na Tabela 1 são apresentadas as informações descritivas das variáveis utilizadas. As que apresentaram maior dispersão relativa, mensurada pelo coeficiente de variação, foram a densidade populacional $(5,25)$ seguida do crescimento populacional $(2,15)$. Essas variáveis, portanto, potencialmente podem gerar a maior disparidade entre municípios no que diz respeito à capacidade de prover serviços públicos para um mesmo nível de carga tributária. 
Tabela 1 - Estatísticas descritivas das variáveis incluídas nos modelos de regressão

\begin{tabular}{lllllll}
\hline & Mínimo & Máximo & Média & Coefic. Var. & Skewness & Kurtosis \\
\hline Cresc. & -1 & 1 & 0,03 & 2,15 & 1,12 & 7,60 \\
Ext. pobr. & 0 & 67 & 13,47 & 0,96 & 1,01 & 0,10 \\
Densid. & 0 & 13025 & 112 & 5,25 & 13,42 & 220,44 \\
Idade 60 & 3 & 29 & 12 & 0,27 & 0,26 & 0,56 \\
Idade 0 a 14 & 7 & 51 & 25 & 0,19 & 0,71 & 1,07 \\
Analfab. & 1 & 44 & 16 & 0,62 & 0,64 & $-0,70$ \\
Renda & 145 & 2129 & 556 & 0,45 & 0,80 & 1,18 \\
PIB & 2708 & 469964 & 15217 & 1,04 & 9,03 & 176,20 \\
FPM & 12 & 5631 & 708 & 0,72 & 2,29 & 8,12 \\
ICMS & 8 & 7956 & 386 & 1,09 & 5,86 & 70,39 \\
SUS & 0 & 1019 & 134 & 0,53 & 2,27 & 15,18 \\
Comp. financ. & 0 & 13421 & 47 & 5,48 & 30,85 & 1412,21 \\
Fundeb & 0 & 1158 & 348 & 0,41 & 0,65 & 0,84 \\
Volunt. & 0 & 4654 & 156 & 1,27 & 5,87 & 79,58 \\
Tranf. legais & 278 & 15727 & 1828 & 0,50 & 3,13 & 22,24 \\
Bolsa Fam. & 0 & 367 & 133 & 0,60 & 0,24 & $-1,23$ \\
\hline
\end{tabular}

Fonte: Elaboração própria.

Quanto às transferências, as que apresentam maior coeficiente de variação são as compensações financeiras $(5,48)$ e as transferências voluntárias $(1,27)$. As compensações financeiras per capita contemplam apenas os municípios exploradores de recursos naturais, criando uma elevada variabilidade nesse item. A variabilidade nas transferências voluntárias sugere que o espaço discricionário favorece a maior heterogeneidade no repasse, enquanto as transferências legais tendem a realizar uma distribuição mais homogênea em termos per capita. 0 Fundeb, por exemplo, é distribuído de acordo com critérios ligados ao número de matrículas na rede pública, que, por sua vez, está diretamente associado com o tamanho da população. Por esse motivo, o Fundeb per capita tende a atingir valores mais constantes entre os municípios. As demais transferências legais também têm, em geral, bastante relação com a população, e, por isso, acabam apresentando coeficientes de variação menores.

Os valores de skewness e kurtosis sugerem a importância do modelo loglinear, que é utilizado complementarmente na análise. A inclusão de muitas variáveis com distribuição muito distante da normal pode tornar difícil a obtenção de resíduos normais no modelo de regressão. Vale destacar que, graficamente, os formatos da distribuição dos resíduos no modelo linear e loglinear não se afastam intensamente do formato da curva normal, apesar de rejeição da hipótese nula de normalidade pelo teste de kolmogorov-smirnov. 
A análise de multicolinearidade revelou que as variáveis renda e extrema pobreza tiveram valores de VIF de, respectivamente, 8,65 e 7,29. As demais variáveis tiveram esse indicador baixo, sendo a maioria inferior a 5 . Apesar de ter duas variáveis com alta multicolinearidade, a significância das mesmas não foi afetada devido ao grande número de observações, que contrabalança possíveis multicolinearidades. Assim, uma vez que a inflação dos erros-padrão não prejudicou o modelo, e dada a importância teórica das duas variáveis, que são vistas como conceitos distintos pela literatura, decidiu-se mantê-las na análise.

\section{Quadro sintético}

A Tabela 2 evidencia os resultados da regressão com os dados originais.

\section{Tabela 2 - Modelos M1 (linear-linear)}

\section{Variável dependente}

Tranf. legais

(M1)

FPM ICMS Volunt.

\begin{tabular}{lcccc}
\hline $\begin{array}{l}\text { Variáveis } \\
\text { independentes }\end{array}$ & & & & \\
Cresc. & -582.005 & -564.952 & 22.554 & -81.972 \\
& $(192.290)^{* *}$ & $(93.394)^{* *}$ & $(80.804)$ & $(49.021)$ \\
Ext. pobr. & -10.548 & -9.483 & -3.142 & -2.299 \\
& $(1.793)^{* *}$ & $(1.150)^{* *}$ & $(0.636)^{* *}$ & $(0.572)^{* *}$ \\
Densid. & -0.151 & -0.078 & -0.043 & -0.022 \\
& $(0.021)^{* *}$ & $(0.010)^{* *}$ & $(0.008)^{* *}$ & $(0.003)^{* *}$ \\
Idade 60 & 104.670 & 77.157 & 21.994 & 13.073 \\
& $(6.967)^{* *}$ & $(4.210)^{* *}$ & $(2.559)^{* *}$ & $(1.813)^{* *}$ \\
Idade 0 a 14 & 37.345 & 17.902 & 8.393 & 8.660 \\
& $(5.863)^{* *}$ & $(3.781)^{* *}$ & $(2.044)^{* *}$ & $(1.662)^{* *}$ \\
Analfab. & -3.132 & -0.895 & -4.067 & -1.332 \\
& $(2.065)$ & $(1.247)$ & $(0.824)^{* *}$ & $(0.855)$ \\
Renda & -0.490 & -0.448 & -0.045 & -0.019 \\
& $(0.154)^{* *}$ & $(0.070)^{* *}$ & $(0.063)$ & $(0.027)$ \\
PIB & 0.029 & 0.004 & 0.018 & 0.000 \\
& $(0.003)^{* *}$ & $(0.001)^{* *}$ & $(0.002)^{* *}$ & $(0.000)^{* * *}$ \\
Cons & -322.775 & -319.078 & -236.976 & -160.071 \\
R2 & $(242.301)$ & $(150.467)^{* * *}$ & $(89.160)^{* *}$ & $(66.182)^{* * *}$ \\
N & 0.28 & 0.22 & 0.56 & 0.03 \\
\hline & 5,190 & 5,190 & 5,190 & 5,190 \\
\hline
\end{tabular}




\begin{tabular}{lcccc}
\hline \multicolumn{4}{c}{ Variável dependente } \\
& Fundeb & SUS & Comp. financ. & Bolsa Fam. \\
\hline Variáveis & & & & \\
independentes & & & & \\
Cresc. & -4.960 & -78.913 & 170.588 & -100.200 \\
& $(28.026)$ & $(13.101)^{* *}$ & $(80.123)^{* * *}$ & $(7.514)^{* *}$ \\
Ext. pobr. & 2.788 & 0.409 & -0.567 & 1.823 \\
& $(0.325)^{* *}$ & $(0.171)^{* * *}$ & $(0.364)$ & $(0.088)^{* *}$ \\
Densid. & -0.012 & 0.005 & -0.006 & 0.004 \\
& $(0.002)^{* *}$ & $(0.002)^{* * *}$ & $(0.002)^{* *}$ & $(0.001)^{* *}$ \\
Idade 60 & -1.317 & -2.372 & 0.286 & -0.631 \\
& $(1.045)$ & $(0.602)^{* *}$ & $(1.259)$ & $(0.255)^{* * *}$ \\
Idade 0 a 14 & 8.557 & 0.797 & -2.646 & 0.101 \\
& $(1.040)^{* *}$ & $(0.586)$ & $(1.286)^{* * *}$ & $(0.283)$ \\
Analfab. & 2.196 & 1.770 & -0.918 & 2.943 \\
& $(0.385)^{* *}$ & $(0.199)^{* *}$ & $(0.491)$ & $(0.104)^{* *}$ \\
Renda & 0.046 & 0.059 & -0.175 & -0.105 \\
& $(0.019)^{* * *}$ & $(0.010)^{* *}$ & $(0.070)^{* * *}$ & $(0.006)^{* *}$ \\
PIB & 0.001 & -0.000 & 0.004 & -0.000 \\
& $(0.000)^{* *}$ & $(0.000)$ & $(0.002)^{* *}$ & $(0.000)$ \\
Cons & 34.807 & 80.069 & 163.370 & 130.205 \\
N & $(41.940)$ & $(23.523)^{* *}$ & $(56.775)^{* *}$ & $(11.546)^{* *}$ \\
& 0.30 & 0.07 & 0.05 & 0.88 \\
\hline
\end{tabular}

Fonte: Elaboração própria.

A regressão com os dados transformados pela aplicação do logaritmo natural é demonstrada na Tabela 3. 
Tabela 3 - Modelos M2 (log-log)

\begin{tabular}{|c|c|c|c|c|}
\hline \multicolumn{5}{|c|}{ Variável dependente } \\
\hline & Tranf. legais & FPM & ICMS & Volunt. \\
\hline \multicolumn{5}{|c|}{ Independentes } \\
\hline \multirow[t]{2}{*}{ Cresc. } & -0.163 & -0.919 & -0.370 & -0.246 \\
\hline & $(0.123)$ & $(0.161)^{* *}$ & $(0.185)^{* * *}$ & $(0.543)$ \\
\hline \multirow[t]{2}{*}{ Ext. pobr. } & -0.062 & -0.230 & -0.134 & -0.018 \\
\hline & $(0.014)^{* *}$ & $(0.020)^{* *}$ & $(0.018)^{* *}$ & $(0.054)$ \\
\hline \multirow[t]{2}{*}{ Densid. } & -0.095 & -0.213 & -0.130 & -0.216 \\
\hline & $(0.004)^{* *}$ & $(0.006)^{* *}$ & $(0.006)^{* *}$ & $(0.016)^{* *}$ \\
\hline \multirow[t]{2}{*}{ Idade 60} & 0.202 & 0.516 & 0.070 & 0.436 \\
\hline & $(0.023)^{* *}$ & $(0.034)^{* *}$ & $(0.036)$ & $(0.110)^{* *}$ \\
\hline \multirow[t]{2}{*}{ Idade 0 a 14} & -0.302 & -0.499 & -0.389 & 0.025 \\
\hline & $(0.044)^{* *}$ & $(0.062)^{* *}$ & $(0.061)^{* *}$ & $(0.202)$ \\
\hline \multirow[t]{2}{*}{ Analfab. } & 0.062 & 0.211 & -0.031 & -0.072 \\
\hline & $(0.015)^{* *}$ & $(0.023)^{* *}$ & $(0.022)$ & $(0.066)$ \\
\hline \multirow[t]{2}{*}{ Renda } & -0.298 & -0.364 & -0.165 & 0.111 \\
\hline & $(0.026)^{* *}$ & $(0.032)^{* *}$ & $(0.033)^{* *}$ & $(0.085)$ \\
\hline \multirow[t]{2}{*}{ PIB } & 0.307 & 0.103 & 0.732 & 0.116 \\
\hline & $(0.012)^{* *}$ & $(0.014)^{* *}$ & $(0.017)^{* *}$ & $(0.042)^{* *}$ \\
\hline \multirow[t]{2}{*}{ _cons } & 3.106 & 3.880 & 0.810 & 1.147 \\
\hline & $(0.114)^{* *}$ & $(0.155)^{* *}$ & $(0.158)^{* *}$ & $(0.473)^{* * *}$ \\
\hline $\mathrm{R} 2$ & 0.37 & 0.44 & 0.72 & 0.06 \\
\hline $\mathrm{N}$ & 5,190 & 5,190 & 5,190 & 5,190 \\
\hline \multicolumn{5}{|c|}{ Variável dependente } \\
\hline & Fundeb & SUS & Comp. financ. & Bolsa Fam. \\
\hline \multicolumn{5}{|c|}{$\begin{array}{l}\text { Variáveis } \\
\text { independentes }\end{array}$} \\
\hline \multirow[t]{2}{*}{ Cresc. } & 0.664 & -0.391 & 0.773 & -1.338 \\
\hline & $(0.247)^{* *}$ & $(0.307)$ & $(0.439)$ & $(0.125)^{* *}$ \\
\hline \multirow[t]{2}{*}{ Ext. pobr. } & -0.022 & 0.208 & 0.043 & 0.376 \\
\hline & $(0.025)$ & $(0.034)^{* *}$ & $(0.043)$ & $(0.016)^{* *}$ \\
\hline \multirow[t]{2}{*}{ Densid. } & 0.011 & 0.009 & -0.119 & 0.008 \\
\hline & $(0.007)$ & $(0.011)$ & $(0.014)^{* *}$ & $(0.004)^{* * *}$ \\
\hline \multirow[t]{2}{*}{ Idade 60} & 0.048 & -0.100 & 0.359 & 0.114 \\
\hline & $(0.051)$ & $(0.055)$ & $(0.076)^{* *}$ & $(0.041)^{* *}$ \\
\hline \multirow[t]{2}{*}{ Idade 0 a 14} & 0.409 & -0.070 & -0.484 & 0.539 \\
\hline & $(0.084)^{* *}$ & $(0.101)$ & $(0.123)^{* *}$ & $(0.092)^{* *}$ \\
\hline
\end{tabular}




\begin{tabular}{lcccc}
\hline & \multicolumn{3}{c}{ Variável dependente } & \\
& Fundeb & SUS & Comp. financ. & Bolsa Fam. \\
\hline Analfab. & -0.015 & 0.090 & 0.231 & 0.479 \\
& $(0.031)$ & $(0.042)^{* * *}$ & $(0.051)^{* *}$ & $(0.021)^{* *}$ \\
Renda & -0.347 & 0.166 & -0.230 & 0.154 \\
& $(0.037)^{* *}$ & $(0.063)^{* *}$ & $(0.061)^{* *}$ & $(0.020)^{* *}$ \\
PIB & 0.129 & 0.015 & 0.485 & -0.059 \\
& $(0.021)^{* *}$ & $(0.026)$ & $(0.044)^{* *}$ & $(0.010)^{* *}$ \\
Cons & 2.172 & 1.528 & -0.197 & 0.463 \\
& $(0.201)^{* *}$ & $(0.266)^{* *}$ & $(0.314)$ & $(0.189)^{* * *}$ \\
R2 & 0.10 & 0.03 & 0.09 & 0.83 \\
$\mathrm{~N}$ & 5,190 & 5,190 & 5,190 & 5,190 \\
\hline
\end{tabular}

Fonte: Elaboração própria.

Para tornar a disposição dos resultados mais elucidativa, adotou-se a legenda apresentada na Tabela 4 . Adotou-se a mediana dos valores positivos $(0,1365)$ como o cut-off a partir do qual os coeficientes foram classificados entre as quatro categorias; aqueles com valores absolutos acima de 0,1365 foram considerados "fortes" e abaixo deste valor, "fracos". Os coeficientes utilizados foram os padronizados, para viabilizar a comparação entre variáveis distintas.

\section{Tabela 4 - Classificação dos coeficientes padronizados}

\begin{tabular}{ccc}
\hline $\begin{array}{c}\text { Coeficiente padronizado } \\
\text { na regressão }\end{array}$ & $\begin{array}{c}\text { Significado em termos de } \\
\text { equalização }\end{array}$ & Símbolo \\
\hline$|\beta|>0,1365$ & Inequalização forte & | \\
$|\beta|<0,1365$ & Inequalização fraca & $\bullet$ \\
$|\beta|<0,1365$ & Equalização fraca & $\bullet$ \\
\hline$\beta \mid>0,1365$ & Equalização forte & $\bullet$
\end{tabular}

Fonte: Elaboração própria.

A classificação dos coeficientes entre as categorias de equalização e inequalização foi realizada considerando o sentido da relação esperada de uma transferência equalizadora para aquele critério, extraído da teoria. Por exemplo, espera-se, no caso da necessidade fiscal associada à proporção da população em idade escolar, que uma transferência equalizadora privilegie municípios que tenham esse indicador mais elevado, ou seja, o percentual da população em idade escolar deve ter uma relação positiva com o volume transferido. Essa relação, por sua vez, é expressa pelo coeficiente da regressão. 
Sintetizaram-se os resultados das 16 regressões por meio da Tabela 5, apresentando as interpretações dos coeficientes para todos os modelos à luz da equalização fiscal.

\section{Tabela 5 - Síntese dos resultados}

\begin{tabular}{|c|c|c|c|c|c|c|c|c|c|c|c|c|c|c|c|c|}
\hline & \multicolumn{2}{|c|}{$\begin{array}{l}\text { Tranf. } \\
\text { legais }\end{array}$} & \multicolumn{2}{|l|}{ FPM } & \multicolumn{2}{|c|}{ ICMS } & \multicolumn{2}{|c|}{$\begin{array}{l}\text { Comp. } \\
\text { financ. }\end{array}$} & \multicolumn{2}{|c|}{ Fundeb } & \multicolumn{2}{|l|}{ SUS } & \multicolumn{2}{|c|}{ Volunt. } & \multicolumn{2}{|c|}{ Bolsa } \\
\hline & M1 & $\mathrm{M} 2$ & M1 & $\mathrm{M} 2$ & M1 & M2 & M1 & $\mathrm{M} 2$ & M1 & $\mathrm{M} 2$ & M1 & $\mathrm{M} 2$ & M1 & $\mathrm{M} 2$ & M1 & $\mathrm{M} 2$ \\
\hline Cresc. & ळ & & & ळ & & ळ & - & & & $\bullet$ & \% & & & & ஓ & ळ \\
\hline Ext. pobr. & $\infty$ & ळ & ळ & ০ & 。 & ळ & & & $\bullet \bullet$ & & $\bullet$ & $\bullet \bullet$ & ০ & & $\bullet \bullet$ & $\bullet \bullet$ \\
\hline Densid. & - & $\bullet \bullet$ & $\bullet$ & $\bullet \bullet$ & $\bullet$ & $\bullet \bullet$ & $\bullet$ & $\bullet$ & $\bullet$ & & 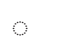 & & - & $\bullet \bullet$ & \% & 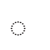 \\
\hline Idade 60 & $\bullet \bullet$ & $\bullet \bullet$ & $\bullet \bullet$ & $\bullet \bullet$ & $\bullet \bullet$ & & & $\bullet$ & & & \% & & $\bullet \bullet$ & $\bullet$ & 。 & - \\
\hline Idade 0 a 14 & $\bullet \bullet$ & $\infty$ & $\bullet \bullet$ & ळ & $\bullet$ & ஓ & \% & \% & $\bullet \bullet$ & $\bullet \bullet$ & & & $\bullet \bullet$ & & & $\bullet \bullet$ \\
\hline Analfab. & & - & & $\bullet \bullet$ & \% & & & $\bullet$ & $\bullet \bullet$ & & $\bullet \bullet$ & - & & & $\bullet \bullet$ & $\bullet$ \\
\hline Renda & $\bullet$ & $\bullet \bullet$ & $\bullet \bullet$ & $\bullet \bullet$ & & $\bullet$ & $\bullet \bullet$ & $\bullet \bullet$ & & $\bullet \bullet$ & $\infty$ & ळ & & & $\bullet \bullet$ & $\infty$ \\
\hline PIB & ळ & চ & \% & ০ & ळ & ০ & $\infty$ & ळ & ळ & ळ & & & \% & 。 & & $\bullet$ \\
\hline
\end{tabular}

Fonte: Elaboração própria.

Nota: As células para as quais o coeficiente não foi significante ao nível de $5 \%$ ficaram vazias.

Os resultados podem ser analisados considerando cada critério separadamente, tendo em vista o impacto de cada transferência em sua equalização, ou, ao contrário, em sua inequalização. A análise a seguir obedece a essa estrutura:

Crescimento: Essa variável corresponde ao crescimento percentual da população do município entre os anos de 2010 e 2007. Alguns autores (BOADWAY, 2007; MENDES; MIRANDA; Cossıo, 2008) destacam o crescimento populacional como uma necessidade fiscal relevante, pois o crescimento exige uma expansão da estrutura de provisão dos serviços públicos. A equalização dessa variável aparentemente não é influenciada de forma intensa pelas transferências, pois não houve nenhuma equalização ou inequalização (termo cunhado neste trabalho para designar o agravamento do hiato fiscal causado por uma transferência) com caráter "forte".

Percentual da população em situação de extrema pobreza: Essa variável equivale ao percentual da população que vive com menos de $\mathrm{R} \$ 70,00$ por mês (valores reais de 2010). Esse indicador reflete uma necessidade fiscal na medida em que implica maiores custos para o governo local na provisão de bens e serviços (MARTINEZVASQUEZ; BOEX, 1999). Essa necessidade é equalizada quando municípios com maior percentual da população em situação de extrema pobreza recebem maiores recursos de transferência. Dentre as transferências intergovernamentais, apenas as transferências de Fundeb e SUS equalizam essa necessidade fiscal. Como FPM e ICMS têm efeito negativo na equalização dessas transferências, ou seja, concedem 
recursos a quem precisa menos nesse quesito, e como essas são as transferências de maior volume, o resultado quando se analisa o total das transferências legais é um forte caráter inequalizador desses recursos no que diz respeito a essa necessidade fiscal. As transferências voluntárias também prejudicam a equalização desse critério, que é contemplado apenas pelo Programa Bolsa-Família, que visa atender justamente essa parcela da população.

Densidade populacional: É um componente importante da necessidade fiscal (BoAdWAY, 2007; Mendes; Miranda; Cossıo, 2008). Neste trabalho, foi utilizada a medida disponibilizada pelo censo 2010, cuja unidade de medida é o número de habitantes por quilometro quadrado $\left(\mathrm{Ha} / \mathrm{Km}^{2}\right)$. Controlados os demais fatores, geralmente municípios com populações esparsas apresentam maiores custos na provisão do serviço público (BLÖCHLIGER, 2013). Os resultados indicam que a maioria das transferências beneficiam municípios com menor densidade populacional. Portanto, pode-se considerar que o quadro geral das transferências, legais e voluntárias, devolutivas ou redistributivas, tem promovido a equalização desse critério.

Percentual da população com idade superior a 60 anos: É outro componente relevante das necessidades fiscais de um governo local (MARTINEZ-VASQUEZ; BOEX, 1999), pois implica maior volume de serviços públicos prestados, principalmente os relacionados à saúde. Os resultados mostram que várias transferências equalizam essa necessidade fiscal. Nesse sentido, municípios com maior percentual de idosos têm recebido mais, tanto do total de transferências legais quanto das transferências voluntárias. Chama atenção, contudo, o fato de que o SUS não beneficia municípios com maior proporção de idosos, apesar de essa faixa etária implicar maiores custos na provisão do serviço de saúde (BLÖCHLIGER, 2013).

Percentual da população com idade entre 0 e 14 anos: Esse indicador reflete os custos associados à provisão da educação básica, sob responsabilidade municipal. Portanto, considera-se existente o efeito equalizador quando essa variável guardar uma relação positiva com o valor transferido (BOADWAY, 2007; MARTINEZ-VASQUEZ; BOEX, 1999; SCHROEDER; SMOKE, 2003). A análise desse critério resultou em sinais opostos entre os modelos M1 e M2 para ICMS, FPM e o total de transferências legais. Portanto, não é possível realizar inferências no papel das transferências de ICMS e FPM nesse quesito, devido à ambiguidade dos coeficientes.

Analfabetismo: Municípios com indicador de analfabetismo elevado apresentam maiores demandas pelo serviço público devido à educação especial (MARTINEZVASQUEZ; BOEX, 1999). Assim, um índice alto de analfabetismo pode refletir a necessidade de realizar investimentos no sistema educacional. $\mathrm{O}$ analfabetismo é uma necessidade fiscal equalizada por todas as transferências, exceto pelas voluntárias, que não têm efeito na equalização, e pelo ICMS, que distribui recursos 
na direção oposta dessa necessidade, considerando nesse último caso, o modelo M2. No quadro geral, o resultado do modelo M1 indica que as transferências legais equalizam essa necessidade, enquanto as voluntárias lhe são indiferentes.

Renda domiciliar per capita: Esse é um dos indicadores de capacidade fiscal utilizados (MARTINEZ-VASQUEZ; BOEX, 1999; SHAH, 2007). A renda familiar reflete uma parte da capacidade do município de gerar receita própria por meio da tributação. Municípios com elevada renda per capita necessitam de menos transferências do que um município com renda menor, considerando os demais fatores constantes (SCHROEDER; SMOKE, 2003). A capacidade fiscal expressa pela renda domiciliar é bastante equalizada. Dentre as transferências intergovernamentais, apenas a do SUS "inequaliza" essa necessidade fiscal, considerando apenas os resultados em que não houve ambiguidades entre os modelos M1 e M2. Portanto, a transferência do SUS beneficia municípios com maior renda domiciliar per capita.

PIB per capita: Municípios com maior PIB per capita detêm maior capacidade de gerar receita tributária, e, por esse motivo, uma transferência equaliza a capacidade fiscal quando privilegia os governos locais com PIB per capita menor (MARTINEZVAsquez; BoeX, 1999; SChroeder; SMOKe, 2003; SHAH, 2007). Esse indicador é inequalizado por todas as transferências intergovernamentais, com exceção do SUS. Assim, quando se analisa o total das transferências legais, percebe-se que o quadro geral beneficia municípios com maior PIB per capita. A transferência do Bolsa Família é a única que promove a equalização dessa capacidade fiscal, quando controlada a renda e as demais necessidades fiscais.

É igualmente elucidativo realizar a análise dos resultados apresentados na Tabela 5 em função das transferências, conforme analisado a seguir:

Transferências legais totais: Os resultados evidenciam que o total de transferências que seguem critérios legais, e, portanto, que não são discricionárias, equaliza a capacidade fiscal atrelada à renda per capita e às necessidades fiscais associadas à densidade populacional e ao percentual de população idosa. Por outro lado, justamente os municípios com maior PIB per capita são privilegiados pelos repasses, não só em virtude da cota-parte do ICMS, que, por definição, é devolutiva e beneficia municípios com maior produção econômica, mas também devido às demais transferências, que, com exceção dos recursos do SUS, tiveram um impacto prejudicial na equalização desse item. O conjunto dessas transferências também intensifica a disparidade fiscal associada ao percentual de população em extrema pobreza, já que quanto pior esse indicador no município, menos o governo local tende a receber essas transferências. Os resultados apontam ainda que a necessidade associada ao crescimento populacional também é inequalizada, devido à participação do ICMS e do FPM, transferências com elevado volume de recursos e que preterem os municípios que cresceram mais nos anos recentes. 
Fundo de Participação dos Municípios: O FPM beneficia municípios que possuem população mais esparsa, com maior índice de analfabetismo, com maior percentual de pessoas com mais de 60 anos e com menor renda domiciliar per capita. Por outro lado, deixa de equalizar critérios importantes, como o PIB, o percentual da população em situação de extrema pobreza e o crescimento populacional. O crescimento é inequalizado porque municípios que crescem podem passar para a faixa subsequente de população, prevista no marco legal e utilizada para distribuir o recurso. Uma vez que, em cada faixa superior de população, o valor per capita recebido dessa transferência é menor, municípios que crescem acabam sendo prejudicados. Além disso, o FPM parte do pressuposto de que municípios menores serão também os que mais necessitam recursos, mas esses não têm necessariamente uma elevada proporção da população em situação de extrema pobreza, e não apresentam PIB per capita sempre pequeno. Pelo contrário, alguns municípios pequenos têm menores necessidades fiscais associadas ao nível de pobreza, assim como possuem capacidade fiscal elevada, associada a um PIB per capita alto. Assim, a transferência contribui para inequalizar esses itens, ou seja, beneficiar municípios com maior PIB per capita, menor proporção da população em extrema pobreza e menor crescimento populacional.

Cota parte do ICMS: Não é pretensão dessa transferência realizar nenhuma forma de equalização fiscal, mas sim devolver os recursos para onde eles foram arrecadados. Sendo um imposto incidente sobre o valor agregado, beneficiar os municípios com maior PIB per capita é uma consequência natural. Além do PIB, esse recurso apresentou caráter inequalizador principalmente para a necessidade fiscal associada ao percentual de população em extrema pobreza. No entanto, apesar de ser uma transferência devolutiva, seus recursos aparentemente têm contribuído para equalizar as necessidades fiscais associadas à densidade populacional e ao percentual da população acima de 60 anos. Vale ressaltar que o papel dessa transferência na equalização é muito similar ao da cota-parte de FPM, apesar dessas transferências terem natureza distintas, e, em certa medida, até opostas. A única diferença significativa é o papel equalizador do FPM no item Analfabetismo, enquanto o ICMS tem um efeito oposto. Dessa forma, aproximadamente $46 \%$ do total de transferências intergovernamentais seguem um mesmo padrão na equalização, pois o ICMS e o FPM, que figuram entre as principais fontes dos recursos municipais, se reforçam mutuamente, na perspectiva da equalização fiscal.

Fundeb: Apesar de não ter diretamente esta função, essa transferência é a que mais promove a equalização fiscal. Apesar de ser uma transferência condicionada, quando essa financia um gasto que iria ser realizado independentemente dessa condicionalidade, ocorre a liberação de recursos próprios para outras áreas (SPAHN, 2007). Com a transferência, o município pode utilizar as receitas próprias para outras 
finalidades, em vez de financiar aquele gasto. Por esse motivo, uma transferência condicionada pode contribuir para o município atender outras necessidades fiscais, além daquelas expressas na condicionalidade.

SUS: É a única transferência, entre as estudadas, que não beneficia municípios com maior PIB per capita. Ainda no quesito capacidade fiscal, um resultado que difere dos obtidos nas demais transferências é o papel inequalizador quando se analisa a renda per capita. No entanto, o SUS desempenha um papel de equalização no que diz respeito às necessidades fiscais associadas ao percentual de analfabetos e população em situação de extrema pobreza.

Cota-parte da compensação financeira: Esse recurso privilegia municípios que cresceram mais, que possuem população mais esparsa, com maior índice de analfabetismo e com maior percentual de pessoas com mais de 60 anos. Essa transferência ainda promove a equalização fiscal ao distribuir menos para os municípios com maior capacidade de gerar arrecadação própria, quando expressa pela renda domiciliar per capita. Contudo, essa transferência privilegia municípios com elevado PIB per capita e com menor percentual de população em idade escolar.

Transferências voluntárias: Essas transferências são distribuídas discricionariamente pelos diferentes níveis de governo, assim não integram o conjunto das transferências legais. Muitos trabalhos indicam que esses recursos têm sido utilizados com fins políticos, beneficiando municípios das bases de apoio do governo transferidor, ou ainda, municípios com elevado número de swing voters (DIXIT; LONDREGAN, 1996; JOHANSSON, 2003), que tomam decisões eleitorais sem critérios ideológicos, mas fundamentadas na provisão de serviços e investimentos. Como outras transferências, inequalizam as necessidades fiscais associadas à população em extrema pobreza e a capacidade fiscal associada ao PIB per capita, contudo, promovem a equalização nos critérios densidade, população acima de 60 anos e população em idade escolar. Entre todas as transferências estudadas, os resultados indicam que essa é a que menos interfere na equalização fiscal, uma vez que 8 dos 16 coeficientes presentes nos dois modelos (M1 e M2) não foram significantemente diferentes de zero.

Bolsa-família per capita: Apesar de não ser intergovernamental, foi investigada neste trabalho devido à sua relevância e ao fato de disputar recursos com outras transferências. Além disso, o volume de recursos pode servir para atenuar ou intensificar o efeito de outras transferências na equalização fiscal. À medida que o recurso privilegia determinados municípios, esse pode atender determinadas necessidades da população e reduzir em algum grau a pressão no orçamento municipal, liberando recursos para serem aplicados em outras áreas. A transferência do Bolsa Família exerce um papel importante na equalização fiscal, equalizando a 
necessidade relacionada ao analfabetismo, à população em situação de extrema pobreza, à população em idade escolar e, em menor grau, à capacidade fiscal expressa pelo PIB. Essa transferência foi a única que desempenhou papel equalizador do PIB entre os municípios, servindo para contrabalancear uma limitação das transferências intergovernamentais. O contrapeso também aconteceu na variável relacionada à proporção da população em situação de extrema pobreza, pois os municípios que têm maior proporção da população nessas condições recebem menos recursos das transferências intergovernamentais, controladas as demais variáveis incluídas nos modelos. Já os recursos do Programa Bolsa Família vão à direção oposta e beneficiam esses municípios, uma vez que essa política visa atender justamente os indivíduos em condições graves de pobreza.

\section{Considerações finais}

O Brasil é um país com território significativamente heterogêneo, em que 5.565 municípios convivem em um mesmo regime federativo, a despeito de suas diferenças econômicas, sociais, culturais e demográficas. Apesar das disparidades entre os municípios, os governos locais receberam maiores atribuições e responsabilidades a partir do processo de descentralização que marcou as últimas décadas. Essa situação criou o desafio de permitir, aos munícipes de diferentes regiões, o acesso a serviços públicos e oportunidades de forma minimamente equitativa. Como as bases tributárias, a partir das quais os municípios podem gerar receitas próprias, bem como as demandas por serviços públicos são distribuídas de maneira desigual ao longo do território, torna-se necessária a equalização fiscal horizontal por meio das transferências intergovernamentais.

Uma vez que, no contexto da equalização fiscal, as transferências podem anular o efeito umas das outras, ou, ao contrário, intensificá-lo, neste trabalho se optou por estudar as transferências em um nível agregado, concomitante ao estudo de cada repasse específico. Numa perspectiva multivariada, também foi analisado como o volume de repasse responde a mudanças em cada necessidade e capacidade fiscal, controlando as demais necessidades e capacidades.

Os resultados mostraram que algumas necessidades fiscais associadas à população em situação de extrema pobreza e crescimento populacional não foram equalizadas pelas transferências legais. Ao contrário, justamente os municípios que menos necessitavam de recursos, segundo esses critérios, foram os que mais receberam, considerando controlada a capacidade de gerar arrecadação própria e as outras demandas. No que diz respeito à capacidade fiscal, o PIB também foi um critério cuja relação com o repasse das transferências legais foi o contrário do 
preconizado pela equalização fiscal, pois justamente os municípios com maior PIB per capita receberam mais.

Dentre as transferências legais, avaliando-as pelo prisma da equalização, o repasse em que se observa a situação mais crítica é o FPM. Os recursos do FPM exercem um impacto negativo na necessidade fiscal associada ao crescimento populacional e população em situação de extrema pobreza, bem como na capacidade fiscal expressa pelo PIB, apesar do objetivo essencialmente redistributivo atrelado a essa transferência. O ICMS também apresenta esses mesmos efeitos, contudo, por ser uma transferência de caráter devolutivo, não se espera que o ICMS tenha resultados positivos para a equalização fiscal. Dessa forma, desperta atenção a similaridade dos efeitos dessas duas transferências na equalização dos diversos itens, uma vez que a cota-parte do FPM tem caráter redistributivo, enquanto a cota-parte do ICMS representa, essencialmente, uma transferência devolutiva. A similaridade significa que ambas se reforçam mutuamente, considerando seus impactos pela perspectiva da equalização.

Por outro lado, os resultados revelam um papel importante do Fundeb na equalização, pois os recursos desse fundo contribuíram para equalizar a maioria das necessidades fiscais. A transferência do SUS também exerceu, em um grau menor, uma função positiva para a equalização. Se, por um lado, é verdade que o SUS apresentou um efeito prejudicial nos critérios de população acima de 60 anos e densidade populacional, por outro, esses critérios já haviam sido amplamente contemplados pelas demais transferências, e, portanto, no cômputo geral, esses itens são equalizados pelas transferências legais. Ao mesmo tempo, a transferência do SUS atua na equalização da necessidade associada à população em situação de extrema pobreza, cujas disparidades são negligenciadas e até agravadas pelas demais transferências.

Apesar do objetivo das transferências condicionais não ser essencialmente promover a equalização fiscal, elas permitem aliviar a pressão orçamentária do governo local. No caso em que o governo iria investir na área da condicionalidade independentemente de qualquer restrição, a transferência condicional tem o mesmo efeito que uma transferência livre de qualquer restrição na aplicação do recurso (SPAHN, 2007).

As transferências voluntárias, por sua vez, foram as que menos interferiram na equalização fiscal dos diversos itens estudados. Esse resultado pode ser explicado pelo fato de que, enquanto repasse discricionário, esses recursos não seguem nem critérios redistributivos, nem devolutivos. Ao contrário, essas transferências são influenciadas por fatores políticos, que podem não ter nenhuma relação com a capacidade ou com a necessidade fiscal do município. 
Outra conclusão importante da pesquisa é que a transferência do Programa Bolsa Família, caso alivie as demandas dos munícipes e libere recursos orçamentários do governo local, pode representar um repasse com contribuição importante para a equalização fiscal. Surpreendentemente essa transferência teve papel equalizador para um elevado número de itens, alguns deles pouco contemplados pelas demais transferências. Por esse motivo, considerando o quadro geral de transferência, o repasse do Programa Bolsa Família pode exercer uma função de contrapeso para aqueles critérios que foram esquecidos pelas transferências intergovernamentais.

Com relação aos critérios, uma conclusão importante é que as disparidades na necessidade fiscal associada ao crescimento populacional e à população em situação de extrema pobreza, bem como as diferenças na capacidade fiscal expressas pelo PIB provavelmente estão sendo excessivamente agravadas pelas transferências. Municípios que cresceram mais ou que apresentaram maior porção da população em situação de pobreza grave receberam menos, para um grande número de transferências, assim como os municípios com PIB per capita mais elevado foram beneficiados pela maioria dos repasses analisados.

Dessa forma, torna-se necessário rever o marco legal que regulamenta os diversos fluxos de recursos que compõem o orçamento no nível local, principalmente no caso de transferências que pretendem justamente realizar a redistribuição de recursos dentro da Federação, como no caso do FPM. Qualquer mudança específica deve ser analisada à luz do quadro geral de transferências, pois, conforme foi observado neste trabalho, é comum uma transferência atenuar, distorcer ou intensificar o efeito de outra, no âmbito da equalização fiscal.

\section{Referências bibliográficas}

ABRÚcIO, F. L. A coordenação federativa no Brasil: a experiência do período FHC e os desafios do governo Lula. Revista de Sociologia Política, v. 24, p. 41-67, jun. 2005.

BACHUR, J. P. Federalismo fiscal, atribuições fiscais constitucionais e equalização regional: EUA, Alemanha e Brasil em perspectiva comparada. Revista do Serviço Público, v. 56, n. 4, p. 377-401, 2005.

BATISTA, M. Burocracia local e qualidade da implementação de políticas descentralizadas: uma análise da gestão de recursos federais pelos municípios brasileiros. Revista do Serviço Público, v. 66, n. 3, p. 345-370, 2015.

BlöCHLIGER, Hansjörg (Ed.). Fiscal Federalism 2014: Making Decentralisation Work. Paris: OECD Publishing, 2013. Disponível em: <http://dx.doi. org/10.1787/9789264204577-en>. Acesso em: 31 ago. 2017.

BOADWAY, R. Grants in a federal economy: a conceptual perspective. In: BOADWAY, $\mathrm{R}$; SHAH, A. (Eds.). Intergovernmental fiscal transfers: principles and practice. Washington, D.C.: World Bank, 2007. 
Cyrenne, P.; PANDEY, M. Fiscal equalization, government expenditures and endogenous growth. International Tax and Public Finance, v. 2, n. 22, p. 311-329, 2015.

DAFFLON, B. Fiscal capacity equalization in horizontal fiscal equalization programs. In: BOADWAY, R; SHAH, A. (Eds.). Intergovernmental fiscal transfers: principles and practice. Washington, D.C.: World Bank, 2007.

Dixit, A.; LondREgAN, J. The determinants of success of special interests in redistributive politics. Journal of Politics, v. 58, p. 659-668, 1996.

Instituto Brasileiro de Geografia e Estatística (IBGE). Censo Demográfico 2000. Rio de Janeiro: IBGE, 2010.

JOHANSSON, E. Intergovernmental grants as a tactical instrument: empirical evidence from Swedish municipalities. Journal of Public Economics, v. 87, p. 883-915, 2003.

MACIEL, P. J.; ANDRADE, J.; TELES, V. K. Transferências fiscais e convergência regional no Brasil. Working Paper. Banco de Nordeste do Brasil, 2008. Disponível em: [http:// edi.bnb.gov.br/content/aplicacao/Eventos/forumbnb2006/docs/transferencias. pdf]. Acesso em: 29 abr. 2016.

Martinez-VAsquez, J.; BoeX, J. The design of equalization grants: theory and applications, Part I: Theory and Concepts. Washington D.C.: The World Bank Institute and AYSPS, Georgia State University, 1999.

MendeS, M.; MIRANDA, R.B.; Cossıo, F. Transferências intergovernamentais no Brasil: diagnóstico e proposta de reforma. Texto para Discussão 40. Brasília: Consultoria Legislativa do Senado Federal, abr. 2008.

ORAIR, R. Esforço fiscal dos municípios: indicadores de condicionalidade para o sistema de transferências intergovernamentais. Monografia premiada em 1 o lugar no XV Prêmio Tesouro Nacional - 2010, Tópicos especiais de finanças públicas. Brasília: Esaf, 2010. 60 p.

Prado, S. (Org.) Competição fiscal. Rio de Janeiro: Fórum Fiscal dos Estados Brasileiros, Caderno n. 2, v. 2, 2006.

Introdução conceitual e visão geral do sistema. In: PrADo, S. (Org.). Transferências intergovernamentais na Federação brasileira: avaliação e alternativas de reforma. Rio de Janeiro: Fórum Fiscal dos Estados Brasileiros, Caderno n. 6, v. 2, 2007.

Transferências fiscais e financiamento municipal no Brasil. Projeto Descentralização Fiscal e Cooperação Financeira Intergovernamental. (Relatório de Pesquisa). Ebap/Fundação Konrad Adenauer, 2001.

RESCHOVSkY, A. Compensating local government for differences in expenditure needs in a horizontal fiscal equalization program. In: BOADWAY, R; SHAH, A. (Eds.). Intergovernmental fiscal transfers: principles and practice. Washington, D.C.: World Bank, 2007.

Rezende, F. Os desafios do federalismo fiscal. In: Rezende, F. (Org.). Desafios do federalismo fiscal. Rio de Janeiro: FGV Editora, 2006. 
Federalismo fiscal: em busca de um novo modelo. In: OLIVEIRA, R. P.; SANTANA, W. Educação e federalismo no Brasil: combater as desigualdades, garantir a diversidade. Brasília: Unesco, 2010. p. 71-88.

SCHROEDER, L.; SMOKE, P. Intergovernmental fiscal transfers: concepts, international practice, and policy issues. In: SMOKE, P.; KIM, Y. H. (Eds.). Intergovernmental transfers in Asia: current practice and challenges for the future. Manila: Asian Development Bank, 2003. p. 20-59.

$\mathrm{SHAH}, \mathrm{A}$. Institutional arrangements for intergovernmental fiscal transfer and a framework for evaluation. In: BOADWAY, R; SHAH, A. (Eds.). Intergovernmental fiscal transfers: principles and practice. Washington, D.C.: World Bank, 2007.

SPAHN, P. B. Intergovernmental transfers: the funding rule and mechanism. In:

WILSON, L. S. Macro formulas for equalization. In: BOADWAY, R; SHAH, A. (Eds.). Intergovernmental fiscal transfers: principles and practice. Washington, D.C.: World Bank, 2007.

WANG, X.; HERD, R. The system of revenue sharing and fiscal transfers in China. OECD Economics Department Working Papers, n. 1030, OECD Publishing, 2013. The system of revenue sharing and fiscal transfers in China. [s.l: s.n.]. Disponível em: <http://dx.doi.org/10.1787/5k4bwnwtmxOr-en>. Acesso em: 30 abr. 2015.

\footnotetext{
Alexandre Lima Baião

Possui doutorado em Administração Pública pela Escola de Administração de Empresas de São Paulo da Fundação Getúlio Vargas (EAESP-FGV). Atualmente é Analista de Planejamento e Orçamento no Ministério do Planejamento, Desenvolvimento e Gestão. Contato: alexandrelimabaiao@gmail.com
}

\section{Armando Santos Moreira da Cunha}

Possui doutorado em Gestão pelo Instituto Superior de Ciências do Trabalho e da Empresa (Portugal). Docente do quadro permanente da Escola Brasileira de Administração Pública e de Empresas (EBAPE-FGV) e da Escola de Direito da Fundação Getúlio Vargas. Contato: armando.cunha@fgv.br

\section{Flávio Sergio Rezende Nunes de Souza}

Possui mestrado em Administração pela Escola Brasileira de Administração Pública e de Empresas (EBAPE-FGV). Atualmente é encarregado das seções de planejamento estratégico e de consultoria na Diretoria de Administração da Marinha (DAdM). Instrutor do Centro de Instrução Almirante Newton Braga (CIANB). Contato: flavio.nunes@ marinha.mil.br 
